\title{
An Orexinergic Projection from Perifornical Hypothalamus to Raphe Pallidus Increases Rat Brown Adipose Tissue Thermogenesis
}

\author{
Domenico Tupone, ${ }^{1}$ Christopher J. Madden, ${ }^{1}$ Georgina Cano, ${ }^{2}$ and Shaun F. Morrison ${ }^{1}$ \\ ${ }^{1}$ Department of Neurological Surgery, Oregon Health \& Science University, Portland, Oregon 97239-3098, and ${ }^{2}$ Department of Neuroscience, University of \\ Pittsburgh, Pittsburgh, Pennsylvania 15260
}

Orexin (hypocretin) neurons, located exclusively in the PeF-LH, which includes the perifornical area (PeF), the lateral hypothalamus (LH), and lateral portions of the medial hypothalamus, have widespread projections and influence many physiological functions, including the autonomic regulation of body temperature and energy metabolism. Narcolepsy is characterized by the loss of orexin neurons and by disrupted sleep, but also by dysregulation of body temperature and by a strong tendency for obesity. Heat production (thermogenesis) in brown adipose tissue (BAT) contributes to the maintenance of body temperature and, through energy consumption, to body weight regulation. We identified a neural substrate for the influence of orexin neurons on BAT thermogenesis in rat. Nanoinjection of orexin-A $(12 \mathrm{pmol})$ into the rostral raphe pallidus $(\mathrm{rRPa})$, the site of BAT sympathetic premotor neurons, produced large, sustained increases in BAT sympathetic outflow and in BAT thermogenesis. Activation of neurons in the PeF-LH also enhanced BAT thermogenesis over a long time course. Combining viral retrograde tracing from BAT, or cholera toxin subunit b tracing from $\mathrm{rRPa}$, with orexin immunohistochemistry revealed synaptic connections to BAT from orexin neurons in PeF-LH and from rRPa neurons with closely apposed, varicose orexin fibers, as well as a direct, orexinergic projection from PeF-LH to rRPa. These results indicate a potent modulation of BAT thermogenesis by orexin released from the terminals of orexin neurons in PeF-LH directly into the rRPa and provide a potential mechanism contributing to the disrupted regulation of body temperature and energy metabolism in the absence of orexin.

\section{Introduction}

Orexins (hypocretins) (de Lecea et al., 1998; Sakurai et al., 1998) are neuropeptides synthesized in the brain exclusively by neurons in the PeF-LH, an area including the lateral hypothalamus $(\mathrm{LH})$, perifornical area $(\mathrm{PeF})$, and lateral parts of the medial hypothalamus. Orexinergic neurons have widespread projections and influence a variety of physiological functions including sleep-wake states and stress-arousal responses (Sakurai et al., 2010; Mieda et al., 2011), and neuroendocrine and autonomic effects (Machado et al., 2002; Smith et al., 2010; Kuwaki, 2011). Loss of orexin neurons from the PeF-LH leads to the disordered sleep patterns of narcolepsy, but is often accompanied by defective energy and metabolic homeostasis, including a high risk for obesity (Kok et al., 2003; Hara et al., 2005) and the potential for altered thermoregulation (Plazzi et al., 2011). The Orx-A isoform has a high affinity for both orexin receptor subtypes $\left(\operatorname{Orx}_{1} R\right.$ and $\left.\operatorname{Orx}_{2} R\right)$ (Sakurai et al., 1998).

\footnotetext{
Received July 29, 2011; revised Sept. 7, 2011; accepted Sept. 20, 2011.

Author contributions: D.T., C.J.M., G.C., and S.F.M. designed research; D.T., C.J.M., G.C., and S.F.M. performed research; D.T., C.J.M., G.C., and S.F.M. analyzed data; D.T., C.J.M., G.C., and S.F.M. wrote the paper.

This work was supported by NIH Grants NS040987 (S.F.M.) and DK082558 (C.J.M.), by an American Heart Association Scientist Development Grant (C.J.M.), and by National Science Foundation Grant NSF-0918867 (G.C.). We thank Jane Igoe and Rubing Xing for their contributions to the histological and immunohistochemical data.

Correspondence should be addressed to Domenico Tupone, Department of Neurological Surgery, Oregon Health \& Science University, 3181 SW Sam Jackson Park Road, Portland, 0R 97239-3098. E-mail: tupone@ohsu.edu.

DOI:10.1523/JNEUROSCI.3909-11.2011

Copyright $\odot 2011$ the authors $\quad 0270-6474 / 11 / 3115944-12 \$ 15.00 / 0$
}

Orexin-containing fibers and orexin receptors are present in several brainstem regions (Peyron et al., 1998; Trivedi et al., 1998; Greco and Shiromani, 2001; Marcus et al., 2001; Ciriello et al., 2003; Berthoud et al., 2005), including the rostral raphe pallidus $(\mathrm{rRPa})$ region of the ventromedial medulla, a principal site of sympathetic and somatic premotor neurons regulating the activity of thermal effectors (Blessing et al., 1999; Morrison, 2011; Nakamura and Morrison, 2011), with a well established role in the regulation of body temperature and in controlling brown adipose tissue (BAT) thermogenesis, energy expenditure and heart rate (Cao and Morrison, 2003; Morrison and Nakamura, 2011). The increase in body temperature following cerebroventricular injection of orexin is accompanied by increased motor activity (Yoshimichi et al., 2001), could be reduced by blocking prostaglandin synthesis (Monda et al., 2001), and increased c-Fos in the raphe pallidus (Berthoud et al., 2005). These sparse observations fail to elucidate the mechanisms or sites of orexin action.

The significant BAT depots in adult humans (Zingaretti et al., 2009) and the recognized significance of BAT thermogenesis in endothermy has lead to increasing debate over the potential for altered BAT energy expenditure to contribute to body weight dysregulation in obesity (Kozak, 2010; Nedergaard et al., 2011). Altered energy homeostasis and the high risk for metabolic syndrome in narcolepsy (Poli et al., 2009), coupled with an influence of the orexin system on sympathetically regulated glucose utilization in skeletal muscle (Shiuchi et al., 2009), stimulated the present investigation of a neural substrate for the influence of 
orexin neurons on BAT thermogenesis and energy expenditure. Conventional and viral retrograde tracing techniques revealed a direct orexinergic projection from PeF-LH to $\mathrm{rRPa}$, targeting rRPa neurons synaptically connected to BAT. Functionally, activation of neurons in PeF-LH or nanoinjections of Orx-A directly into $\mathrm{rRPa}$ elicited a marked and prolonged enhancement of BAT sympathetic outflow and BAT thermogenesis. These results indicate a potent thermogenic role for orexin released from the terminals of PeF-LH neurons to directly activate neurons in the rRPa, likely the local BAT sympathetic premotor neurons.

\section{Materials and Methods}

Experiments were performed in accordance with the Guide for the Care and Use of Laboratory Animals, Eighth Edition (National Research Council, National Academies Press, 2010) and protocols were approved by the Institutional Animal Care and Use Committees of Oregon Health and Science University and of the University of Pittsburgh.

\section{Anatomical studies}

Viral injections and immunohistochemical procedures. Adult male, Sprague Dawley rats (275-350 g, Zivic-Miller Laboratories) were maintained in a $12 \mathrm{~h}$, light/dark cycle (lights on at 7:00 A.M.) with ad libitum access to food and water. Rats were housed in a Biosafety level 2 facility throughout the experiments and their health was carefully monitored after viral injections.

Rats were anesthetized with an intraperitoneal injection of ketamine $(60 \mathrm{mg} / \mathrm{kg})$ and xylazine $(7 \mathrm{mg} / \mathrm{kg})$. The interscapular BAT was exposed via a $2.5 \mathrm{~cm}$ incision along the back, and $0.1 \mu \mathrm{l}$ of an attenuated strain of Pseudorabies virus [PRV-Bartha, $4 \times 10^{8}$ plaque-forming units ( $\mathrm{pfu}$ )/ $\mathrm{ml}$ ] was injected into 5 different sites in a circular distribution (total volume $=0.5 \mu \mathrm{l}$ ), using a $1 \mu \mathrm{l}$ Hamilton syringe. After injections, the skin was closed with wound clips. Different postinoculation times were used to estimate the temporal progression of the virus through the CNS after injection into BAT: $78 \mathrm{~h}$ ( 2 rats), $84 \mathrm{~h}$ (1 rat), $90 \mathrm{~h}$ ( 2 rats) and $96 \mathrm{~h}$ (2 rats). A second group of rats was injected with PRV-BaBlu $\left(1 \times 10^{8}\right.$ $\mathrm{pfu} / \mathrm{ml}$ ), an isogenic recombinant of PRV-Bartha that contains the LAC $\mathrm{Z}$ gene encoding for $\beta$-galactosidase under the control of the viral promoter. As PRV-Bartha, PRV-BaBlu is transported retrogradely through polysynaptic circuits after injection in a specific target (Standish et al., 1995; Elmquist and Saper, 1996; Card and Enquist, 1999; Billig et al., 2000). Following a similar procedure as detailed above, a total volume of $1 \mu \mathrm{l}$ of PRV-BaBlu ( 5 injections of $0.2 \mu \mathrm{l}$ ) was injected into BAT in 6 rats. The inoculation times used were: $84 \mathrm{~h}$ ( 3 rats), $96 \mathrm{~h} \mathrm{(} 2$ rats), and $102 \mathrm{~h} \mathrm{(1}$ rat). After the appropriate post-inoculation time, rats were deeply anesthetized with ketamine $(120 \mathrm{mg} / \mathrm{kg})$ and xylazine $(14 \mathrm{mg} / \mathrm{kg})$ and were perfused transcardially with $0.9 \%$ saline followed by paraformaldehydelysine-periodate fixative. The brains were after fixed for $3 \mathrm{~h}$ and preserved in $25 \%$ sucrose at $4^{\circ} \mathrm{C}$. Serial coronal sections $(35 \mu \mathrm{m})$ were cut with a freezing-stage microtome, collected sequentially in 8 sets and stored in cryoprotectant solution at $-20^{\circ} \mathrm{C}$ before immunohistochemical processing. The tissue samples from rats injected with PRV-Bartha used in this study are a subset of infection cases from a previous study in which the temporal distribution of PRV-infected neurons in the CNS after BAT injection was described in detail (Cano et al., 2003).

A dual fluorescence labeling procedure was performed in one set of brain sections from each rat to characterize PRV-positive (Bartha or $\mathrm{BaBlu}$ ) and orexin-positive neurons. Neurons infected with PRV-Bartha were identified using a rabbit polyclonal antiserum $(\mathrm{Rb} 133,1: 2000)$ that recognizes viral envelope and capsid proteins (Card and Enquist, 1994). Neurons infected with PRV-BaBlu were labeled using a mouse anti- $\beta$ galactosidase antibody $(\mathrm{m} \alpha \beta$-gal, Sigma, 1:1000). A goat polyclonal antibody against Orx-A (gt $\alpha$ Orx, Santa Cruz Biotechnology, 1:1000) was used to identify orexinergic neurons in the PeF-LH. Brain sections were pretreated with $0.5 \%$ sodium borohydride in $10 \mathrm{~mm}$ sodium phosphate buffer (PBS) during 30 min to reduce protein cross-linking caused by aldehyde fixation. Subsequently, sections were incubated for $1 \mathrm{~h}$ at room temperature followed by $48 \mathrm{~h}$ at $4^{\circ} \mathrm{C}$ in a mixture of two primary antibodies (gt $\alpha$ Orx and Rb 133 for PRV-Bartha injections, and gt $\alpha$ Orx and $\mathrm{m} \alpha \beta$-gal for PRV-BaBlu injections) diluted in PBS containing $1 \%$ normal donkey serum and $0.3 \%$ Triton X-100. Then, sections were incubated for $2 \mathrm{~h}$ in the dark at room temperature in a combination of two secondary antibodies: Alexa Fluor 555-conjugated donkey anti-goat IgG (1:500; Invitrogen) to label the cytoplasm of Orx-A-containing neurons and projections in red and DyLight 488-conjugated donkey anti-rabbit IgG (1:500; Jackson ImmunoResearch Laboratories) or Alexa Fluor 488conjugated donkey anti-mouse IgG (1:500; Invitrogen) to label the nucleus (early infection) or the nucleus and cytoplasm of PRV-Bartha- and PRV-BaBlu-infected neurons, respectively, in green. Double-labeled, PRV-infected, Orx-A-immunoreactive (Orx-A-ir) neurons displayed yellow fluorescence in the cytoplasm. All steps were preceded by several washes in PBS. Subsequently, sections were mounted, air-dried, dehydrated and cleared in xylene and coverslipped.

Tissue analysis. The neuroanatomical location of PRV-infected neurons in $\mathrm{rRPa}$, parapyramidal area (PaPy), and of Orx-A-ir neurons in the PeF-LH was based on the stereotaxic rat brain atlas of Paxinos and Watson (Paxinos and Watson, 2007). PRV-infected neurons (non-Orx-A-ir) and PRV-infected, Orx-A-ir neurons (dual-labeled neurons) were counted in the PeF-LH bilaterally in 5 consecutive sections (from a 1:8 set of sections; $245 \mu \mathrm{m}$ distance between consecutive sections). The percentages of PRV-infected, Orx-A-ir neurons relative to the total number of infected neurons in the entire PeF-LH were calculated for each rat. Cases were grouped based on the total number of PRV-infected neurons in the PeF-LH (see Results) and the mean \pm SEM were calculated for each group. Photomicrographs of PRV-infected neurons and Orx-A-ir neurons and fibers were obtained using an image capture system (Simple PCI, version 6.6, Hamamatsu Corp.) attached to an Olympus BX51 fluorescence microscope. The photomicrographs were assembled into a plate using Adobe Photoshop to adjust contrast and brightness without altering the original colors.

$C T b$ injections in $\mathrm{rRPa}$ and PaPy and immunohistochemical procedures. Four adult male, Sprague Dawley rats $(240-400 \mathrm{~g})$, anesthetized with 3\% isoflurane in $100 \% \mathrm{O}_{2}$, were stereotaxically injected with cholera toxin subunit $\mathrm{b}(\mathrm{CTb}, 1 \mathrm{mg} / \mathrm{ml}, 120 \mathrm{nl})$ conjugated to Alexa488 either into $\operatorname{rRPa}(n=2)$ or PaPy $(n=2$; coordinates with incisor bar at $-11 \mathrm{~mm}: 3.0$ $\mathrm{mm}$ rostral, $1.1 \mathrm{~mm}$ lateral, and $3.0 \mathrm{~mm}$ ventral to calamus scriptorius) and the pipette was left in place for $5 \mathrm{~min}$. Rats were treated with antibiotic $(40,000 \mathrm{U} / \mathrm{kg}$ penicillin $\mathrm{G}$, i.m. $)$, analgesic $(0.05 \mathrm{mg} / \mathrm{kg}$ buprenorphine, i.m.) and saline ( $3 \mathrm{ml}$, s.c.). After $7 \mathrm{~d}$, the rats were deeply anesthetized with pentobarbital $(80 \mathrm{mg} / \mathrm{kg}$, i.p.) and transcardially perfused with saline followed by $4 \%$ paraformaldehyde. The brains were postfixed in $4 \%$ paraformaldehyde for $1-2 \mathrm{~h}$ and equilibrated overnight in PBS with $20 \%$ sucrose and $0.01 \%$ sodium azide. Serial coronal sections $(30 \mu \mathrm{m})$ were cut with a freezing-stage microtome, collected sequentially in 5 sets and stored in PBS with $0.01 \%$ sodium azide at $4^{\circ} \mathrm{C}$.

Sections containing PeF-LH were preincubated in an antibody dilution solution (ADS: $500 \mathrm{ml}$ of PBS, $0.3 \%$ Triton X-100, $1.25 \mathrm{~g}$ of carrageenan, $100 \mathrm{mg}$ of $\mathrm{NaN}_{3}, 5 \mathrm{ml}$ of normal donkey serum) for $3 \mathrm{~h}$, pretreated with avidin-biotin blocking kit (SP-2001, Vector) and incubated overnight at room temperature with the primary antibodies for Orx-A (1:5000, rabbit-anti-orexin-A, PC362, Calbiochem) and CTb (1: 20,000, goat-anti-CTb, 703, List Biological). After two washes in PBS containing $0.3 \%$ Triton X-100 (TPBS, $20 \mathrm{~mm}$ ), the tissue was incubated for $1 \mathrm{~h}$ in ADS containing in the secondary antibody for Orx-A (1:500, biotin-SP-donkey-anti-rabbit, Jackson ImmunoResearch Laboratories). After two washes in TPBS, the tissue was incubated for $1 \mathrm{~h}$ in ADS containing the secondary antibody for CTb $(10 \mu \mathrm{g} / \mathrm{ml}$, Alexa Fluor 488 donkey-anti-goat, Invitrogen) and streptavidin conjugated to Alexa Fluor 594 (3-5 $\mu \mathrm{g} / \mathrm{ml}$, Invitrogen). After fluorescent labeling, the tissue was washed in PBS and mounted onto coated slides, air dried and coverslipped with anti-fade mounting medium (Pro-Long Gold, Invitrogen).

Tissue analysis. The neuroanatomical designations of the injection sites in $\mathrm{rRPa}$ and PaPy and those of CTb- and Orx-A-labeled neurons in the PeF-LH are based on the stereotaxic rat brain atlas of Paxinos and Watson (Paxinos and Watson, 2007). Photomicrographs of brain sections and labeled neurons were taken with a camera attached to an Olympus BX51fluorescence microscope. The photomicrographs were 
assembled into a plate using Adobe Photoshop to adjust contrast and brightness without altering the original colors.

\section{Physiological experiments}

Surgical procedures. Male Wistar rats (300-400 $\mathrm{g}$, Charles River, $n=30$ ) were maintained in a $12 \mathrm{~h}$, light/dark cycle (light on at 7:00 A.M.) with ad libitum access to food and water. Rats were anesthetized initially with $3 \%$ isoflurane in $100 \% \mathrm{O}_{2}$ and transitioned to urethane $(0.8$ $\mathrm{g} / \mathrm{kg}$ ) and chloralose $(80 \mathrm{mg} / \mathrm{kg}$ ) following cannulation of a femoral artery and vein. Heart rate (HR) was derived from the arterial pressure signal. After the animals were positioned prone in a stereotaxic frame with the incisor bar at $-4 \mathrm{~mm}$ and with a spinal clamp on the T10 vertebra, they were paralyzed with D-tubocurarine $(0.3 \mathrm{mg}$ initial dose, $0.1 \mathrm{mg} / \mathrm{h}$ supplements) and artificially ventilated via a tracheal cannula with $100 \% \mathrm{O}_{2}(60$ cycles $/ \mathrm{min}$, tidal volume $3-4.5 \mathrm{ml})$. Small adjustments in minute ventilation were made as necessary to maintain basal mixed-expired $\mathrm{CO}_{2}$ levels between 3.0 and $4.5 \%$. Thermocouples, connected to a thermocouple meter (TC-2000, Sable Systems) to obtain analog voltage signals, were placed on the shaved abdominal skin to measure skin temperature $\left(\mathrm{T}_{\text {Skin }}\right)$ beneath the water jacket and $6 \mathrm{~cm}$ into the rectum to measure core temperature $\left(\mathrm{T}_{\text {Core }}\right)$ and into the medial aspect of the left interscapular BAT (iBAT) pad to measure BAT temperature $\left(\mathrm{T}_{\mathrm{BAT}}\right)$. The surgical procedure to insert the thermocouple into the left iBAT pad and, subsequently, to dissect a sympathetic nerve (see below) entering the right iBAT pad involves a midline incision through the back skin and a midline division of the iBAT pad, which leaves both halves of the iBAT pad partially exposed to the ambient temperature. This procedure results in a $\mathrm{T}_{\mathrm{BAT}}$ that, while an accurate measure of the temperature in the left iBAT pad, would be lower than the temperature of other BAT pads. Thus, while it is a useful monitor of the overall changes in BAT thermogenesis, it could be, unexpectedly lower than $\mathrm{T}_{\text {Core }}$, not only at baseline, but also during experimental procedures that increase both BAT thermogenesis and $\mathrm{T}_{\text {Core }}$, when thermodynamics would indicate that $\mathrm{T}_{\mathrm{BAT}}$ should be greater than $\mathrm{T}_{\text {Core }}$ to effect heat transfer. $\mathrm{T}_{\text {Core }}$ was maintained at $\sim 37^{\circ} \mathrm{C}$ with a water jacket continuously perfused with warm water. As required for specific experimental protocols, $\mathrm{T}_{\text {Skin }}$ and $\mathrm{T}_{\text {Core }}$ were adjusted by changing the temperature of the water perfusing the thermal jacket: cooling elicited a basal sympathetic nerve activity to BAT (BAT SNA) and warming inhibited BAT SNA via stimulation of skin thermal receptors (Nakamura and Morrison, 2008).

Postganglionic BAT SNA was recorded from the central cut end of a small nerve bundle dissected from the ventral surface of the right interscapular BAT pad after dividing the fat pad along the midline and reflecting it laterally. Nerve activity was recorded with bipolar hook electrodes, filtered (1-300 Hz), and amplified (20,000×; Cyberamp 380, Molecular Devices). Relative to lambda, the coordinates for the rRPa were anteroposterior $-3.0 \mathrm{~mm}$, mediolateral $0.0 \mathrm{~mm}$, dorsoventral -9.5 to -9.8 $\mathrm{mm}$ below the dural surface, with the optimal dorsoventral site for nanoinjections into $\mathrm{rRPa}$ being that yielding the lowest microstimulation threshold $(<10 \mu \mathrm{A})$ for evoking an excitatory BAT SNA potential.

Nanoinjections. Glass micropipettes (outer tip diameter, 20-30 $\mu \mathrm{m}$ ) were used for all drug nanoinjections which were given over a 5-10 s, using a pressure injection system (model IIe, Toohey Company). Nanoinjection volumes $(60 \mathrm{nl})$ were determined from the displacement of the fluid meniscus in the micropipette. To make multiple nanoinjections at the same site, the micropipette was retracted vertically, emptied, rinsed
Table 1. PRV-infected neurons and orexin-immunoreactive neurons in PeF-LH

\begin{tabular}{lcll}
\hline Infection group & $\begin{array}{l}\text { Number of PRV-infected } \\
\text { neurons in PeF-LH }\end{array}$ & $\begin{array}{l}\text { Number } \\
\text { of rats }\end{array}$ & $\begin{array}{l}\text { Orexin }+\mathrm{PRV}^{+} \text {/ } \\
\text { total PRV }\end{array}$ \\
\hline Light infection & $1-4$ & 2 & $0 \%$ \\
Intermediate infection & $2-8$ & 3 & $44.0 \pm 5.5 \%$ \\
High infection & $10-65$ & 5 & $42.0 \pm 10.2 \%$ \\
\hline
\end{tabular}

with saline, refilled and then repositioned to the original dorsoventral coordinate. The microinjection sites were marked by pressure microinjection of fluorescent polystyrene microspheres (1:10 dilution of FluoSpheres, F8797, F8801 or F8803, Invitrogen). After physiological recordings, rats were perfused transcardially with $0.9 \%$ isotonic saline, followed by $4 \%$ paraformaldehyde in PBS. The brains were removed, postfixed ( $2 \mathrm{~h}$ ), equilibrated overnight in $20 \%$ sucrose and $0.01 \%$ sodium azide and sectioned ( $30 \mu \mathrm{m}$ coronal sections) to localize the fluorescent spots indicating the centers of the injection sites. In some rats, brain sections containing injection sites in PeF-LH were processed for immunohistochemical labeling for Orx-A (see below) to determine the relationship of the injection site to the location of orexinergic neurons. All the coordinates used for the brain intraparenchymal injections were adapted from the atlas of Paxinos and Watson (Paxinos and Watson, 2007).

Drugs. Orexin-A, N-methyl-D-aspartate (NMDA) and the orexin antagonist, SB-334867, were obtained from Tocris Bioscience and dissolved in isotonic saline, except SB-334867 that was dissolved in dimethyl sulfoxide (DMSO) and diluted in isotonic saline to a final concentration of $<10 \%$ DMSO. Orx-A was chosen for these studies based on its high affinity for both $\mathrm{Orx}_{1}-\mathrm{R}$ and $\mathrm{Orx}_{2}-\mathrm{R}$ and the dose was based on a previous study using direct intraparenchymal injections (Chen et al., 2000). The 
Ai

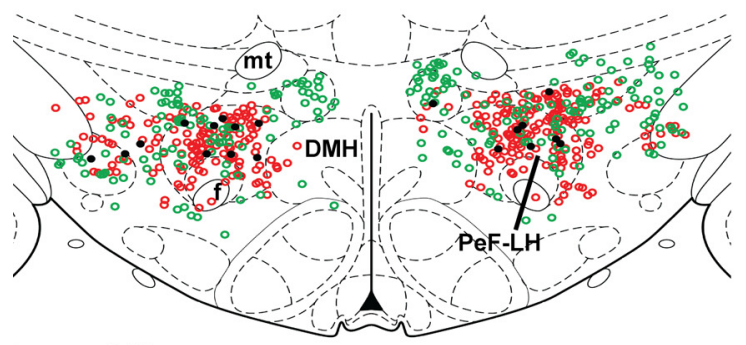

bregma $-3.00 \mathrm{~mm}$

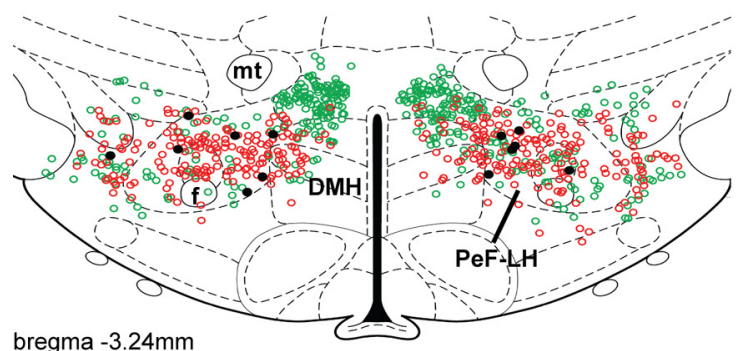

bregma $-3.24 \mathrm{~mm}$

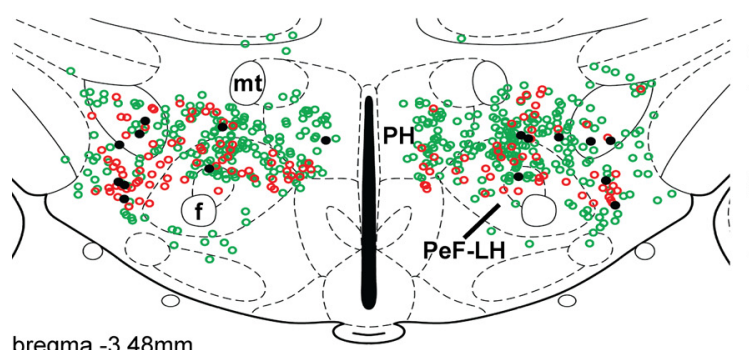

bregma $-3.48 \mathrm{~mm}$

- CTb

Aii
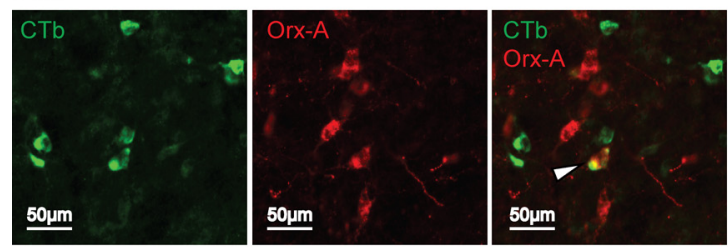

Aiii

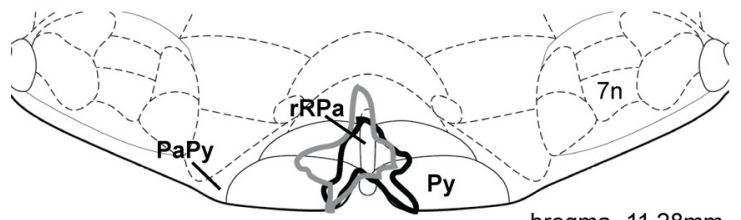

$\mathrm{Bi}$

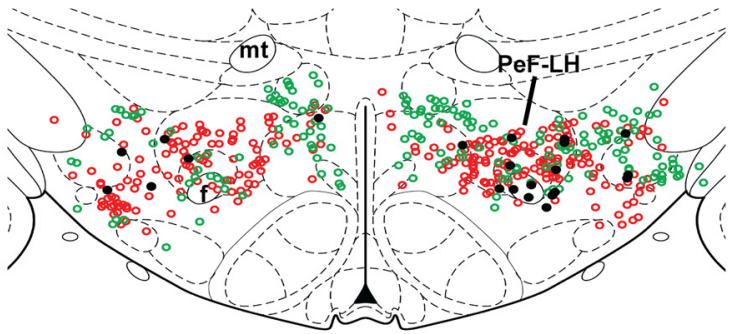

bregma $-3.00 \mathrm{~mm}$

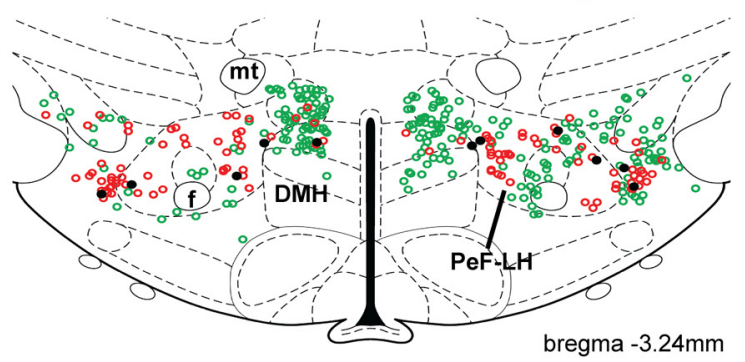

bregma $-3.24 \mathrm{~mm}$

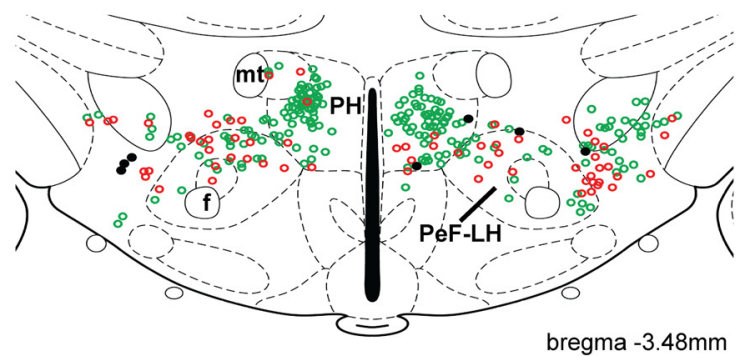

bregma $-3.48 \mathrm{~mm}$

\section{Bii}
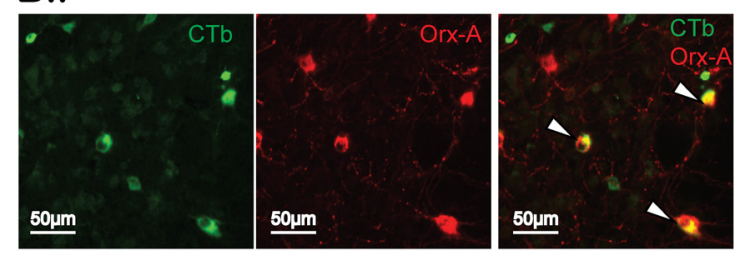

Biii

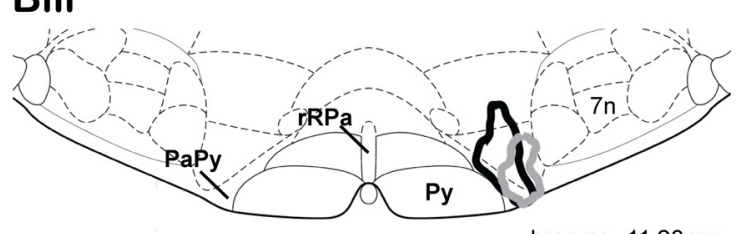

Figure 2. Orx-A-ir neurons in the PeF-LH were retrogradely labeled following CTb injection into the rRPa and into the PaPy. Schematic diagrams in Ai and Bi show the locations and distribution of (Tb-containing (green circles), Orx-A-ir (red circles) and double-labeled (black dots) neurons following (Tb injections into rRPa (Aiii) and into PaPy (Biii), respectively, plotted on atlas drawings (Paxinos and Watson, 2007) at 3 rostrocaudal levels of the PeF-LH. The photomicrographs in Aii and Bii show examples of double-labeled neurons (arrowheads) in PeF-LH following (Tb injections in rRPa and in PaPy, respectively. 7n, Facial nucleus; f, fornix; mt, mammillary tract; PH, posterior hypothalamus; Py, pyramidal tract.

concentration of SB-334867 was based upon the effective dose of orexin, the relative affinities for orexin receptors and the previous observation that the concentration of SB-334867 required to block ( $>90 \%$ inhibition) Orx-A-evoked $\mathrm{Ca}^{2+}$ entry was 100 -fold higher than that of Orx-A (Smart et al., 2001).

\section{Experimental protocols}

Experiment 1: injection of Orx-A in $r R P a$. Rats $(n=7)$ receiving a nanoinjection of Orx-A (12 pmol) into $\mathrm{rRPa}$ at baseline conditions in which $\mathrm{T}_{\text {Core }}$ was maintained at a temperature slightly below $37^{\circ} \mathrm{C}$, which produced a low level of basal BAT SNA.
Experiment 2: injection of Orx-A in the PaPy. Rats $(n=4)$ received a nanoinjection of Orx-A (12 pmol) into PaPy (left side; coordinates: $3.0 \mathrm{~mm}$ caudal to lambda, $1.2 \mathrm{~mm}$ lateral to the midline, and $9.5-9.8 \mathrm{~mm}$ ventral to the dura) under the same baseline cool conditions as in Experiment 1.

Experiment 3: injection of Orx- $A$ in $r R P a$ in warm animals. Rats $(n=4)$ received a nanoinjection of Orx-A (12 pmol) into $\mathrm{rPa}$ while $\mathrm{T}_{\text {Core }}$ was maintained at a sufficiently warm temperature $\left(>37^{\circ} \mathrm{C}\right)$ to ensure the absence of spontaneous BAT SNA.

Experiment 4: injection of NMDA in PeF-LH. Rats $(n=6)$ received a nanoinjection of NMDA (12 pmol) into the PeF-LH (coordinates: 
$3.0 \mathrm{~mm}$ caudal to bregma, $1.2 \mathrm{~mm}$ lateral to the midline, and $8.0-8.4 \mathrm{~mm}$ ventral to dura) under the same baseline cool conditions as in Experiment 1.

Experiment 5: injection of NMDA in PeF-LH in warm animals. Rats $(n=4)$ received a nanoinjection of NMDA (12 pmol) into the PeF under the same warm-temperature baseline conditions as in Experiment 3.

Experiment 6: injection of SB-334867 in $\mathrm{rRPa}$. Rats $(n=7)$ received a nanoinjection of SB334867 ( 3 nmol, $n=5$; 6 pmol, $n=2$ ) into rRPa under the same baseline cool conditions as in Experiment 1. Nanoinjections of vehicle (10\% DMSO) were performed before and after SB-334867 injection.

\section{Data and statistical analyses}

BAT SNA, $\mathrm{T}_{\mathrm{BAT}}, \mathrm{T}_{\text {Core }}, \mathrm{T}_{\text {Skin }}$, expired $\mathrm{CO}_{2}$, arterial pressure, and stimulus trigger pulses were digitized [Micro 1401 MKII; Cambridge Electronic Design (CED)] and recorded onto a computer hard drive for subsequent analysis (Spike 2, CED). In each protocol, baseline was recorded for $10 \mathrm{~min}$, and the physiological changes induced by the drug injection were followed for at least $1 \mathrm{~h}$ unless otherwise noted. Spike 2 software (CED) was used to obtain a continuous measure (with $4 \mathrm{~s}$ bins) of BAT SNA amplitude. This was accomplished by calculating the root mean square (rms) value of the BAT SNA (square root of the total power in the $0.1-20 \mathrm{~Hz}$ band) from the autospectra of sequential $4 \mathrm{~s}$ segments of BAT SNA. For examining the effects of drug treatments under cool conditions evoking a low level of basal BAT SNA, baseline (1 min before drug injection) and peak levels of BAT SNA are normalized to the control level of BAT SNA, taken as the mean BAT SNA amplitude during a 2 min period of minimum BAT SNA recorded when the rat was in a warm condition $\left(\mathrm{T}_{\text {Core }}>37^{\circ} \mathrm{C}\right)$ and basal BAT SNA was absent.

All statistics were performed using Systat software (Version 10, Cranes Software International). Data in the text, figures and table are expressed as mean \pm SEM. Statistical significance was assessed using a paired $t$ test after testing data for normality. For each variable, statistical comparisons were done between the $1 \mathrm{~min}$ baseline period before the injection and the 1 min window at the peak or nadir of the treatment-evoked effect. Due to the short duration of the response after the orexin nanoinjection in Experiments 5 and 6 , a $30 \mathrm{~s}$ window was used for the statistical comparison. Statistic comparison of the effect of orexin injection in rRPa versus NMDA injection in PeF-LH was performed by two-way ANOVA with repeated measures. Statistical results with $p<0.05$ were considered significant.

\section{Results}

\section{PRV injections into BAT}

To determine whether orexin neurons in PeF-LH might be synaptically connected to rRPa neurons that regulate BAT thermogenesis, we injected PRV, which is exclusively retrogradely transported through synapses, into BAT and assessed, simultaneously, the presence of PRV-infected orexin neurons in the PeF-LH and of orexin fibers in close apposition to PRV-infected neurons in the rRPa and PaPy. Importantly, the presence of PRV-infec-

ted neurons in PeF-LH and rRPa was determined at a series of post-inoculation times following PRV injections into BAT to provide an estimation of the relative sequence of retrograde infections of neurons in these two regions.

The pattern of infection in the CNS was reproducible and almost identical after PRV-Bartha or PRV-BaBlu injections into BAT. Since both viruses are retrogradely transported only through synaptically linked neurons, the infected brain regions are most likely those involved in the control of sympathetic outflow to BAT. As in our earlier detailed description of PRV-Bartha infection in the CNS after BAT injection (Cano et al., 2003), infection was first detected in the sympathetic preganglionic neurons (SPNs) located in the intermediolateral cell column of the upper thoracic cord at early post-inoculation times. At longer times, the infection progressed within the spinal cord and into the brain, where infected neurons were observed in sympathetic premotor areas, including the medullary raphe nuclei, containing the rRPa. These brain neurons most likely became infected via direct projections to infected SPNs.

At short post-inoculation times, a few infected neurons were observed in the intermediate extent of the caudal raphe nucleus, mainly in the RMg and $\mathrm{rRPa}$. At intermediate post-inoculation times, infected raphe neurons were found between -10.30 to $-12.30 \mathrm{~mm}$ from bregma (Paxinos and Watson, 2007), being most numerous between -11.00 and $-11.60 \mathrm{~mm}$ from bregma (Fig. 1 $A, B$ ). Rostrally, PRV-infected neurons were found in the $\mathrm{RMg}, \mathrm{rRPa}$ and PaPy; more caudally, PRV-infected neurons were 

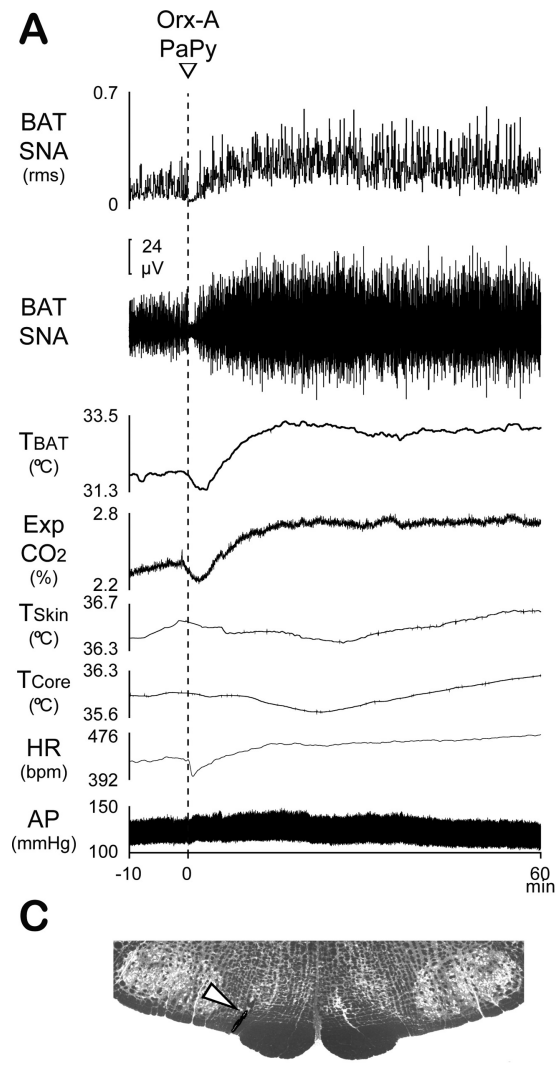
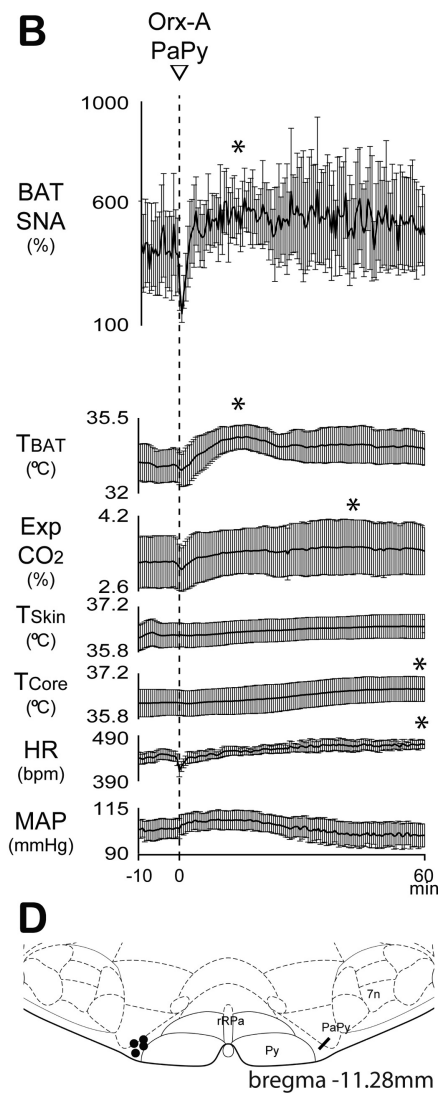

Figure 4. A, Under the baseline condition of a low level of BAT SNA, elicited by skin and core cooling, nanoinjection of Orx-A (dashed line) in the lateral PaPy elicited a marked and sustained $(>1 \mathrm{~h})$ enhancement of BAT SNA, $\mathrm{T}_{\mathrm{BAT}}$, and $\operatorname{expired} \mathrm{CO}_{2}\left(\operatorname{Exp}_{\mathrm{CO}_{2}}\right)$, as well as an increase in $\mathrm{T}_{\text {Core }}$ and HR. Orx-A did not change mean arterial pressure (MAP). The means $\pm \mathrm{SEM}(n=4)$ of the time courses of the physiological variables (points are 30 s averages of the variable values) during the nanoinjection of $0 \mathrm{rx}-\mathrm{A}$ into $\mathrm{PaPy}$ are shown in $\boldsymbol{B} .{ }^{*} p<0.05$, significant increases, comparisons of average variable values during 1 min windows before 0 rx-A injection vs $1 \mathrm{~min}$ at the peak effect. $\boldsymbol{C}$, Histological section shows the Orx-A nanoinjection site in PaPy (white arrowhead) for the responses illustrated in $\boldsymbol{A}$. D. The distribution of Orx-A nanoinjection sites are plotted on an atlas drawing (Paxinos and Watson, 2007) containing the PaPy at the level of the facial nucleus (7n). Py, Pyramidal tract.

observed in the rRPa, raphe obscurus (ROb) and $\mathrm{PaPy}$, although the number of infected neurons was much lower than in rostral sections. At longer post-inoculation times, PRV-infected neurons were observed in all sections throughout the rostrocaudal extent of the medullary raphe nuclei.

Dual fluorescence labeling suggested that PRV-infected neurons in the $\mathrm{rRPa}$ and $\mathrm{PaPy}$ were heavily innervated by orexin fibers (Fig. $1 A, B$ ): apposition-like structures were observed at high magnification in both regions but appeared denser in the PaPy (Fig. 1C). At short post-inoculation times, when only sympathetic premotor groups would have been infected, orexin fibers were observed in close apposition to infected neurons in $\mathrm{rRPa}$ and $\mathrm{PaPy}$, consistent with orexin fibers synapsing on BAT sympathetic premotor neurons in $\mathrm{rRPa}$ and $\mathrm{PaPy}$ that directly project to the spinal cord. At longer post-inoculation times that allowed for another viral replication cycle to occur, orexin fibers apposing infected neurons in $\mathrm{rRPa}$ and $\mathrm{PaPy}$ (Fig. $1 A-C$ ) were most likely innervating interneurons within the raphe nuclei that became infected via their local inputs to infected BAT sympathetic premotor neurons. Although varicose orexin fibers in close apposition to infected $\mathrm{rRPa}$ and PaPy neurons have been previously reported after PRV injection into BAT (Berthoud et al., 2005), the large extent of infection in this study precluded their opportunity to suggest that such orexin fibers may have directly innervated BAT sympathetic premotor neurons.
PRV-infected neurons in the PeF-LH appeared later than those in the medullary raphe nuclei and reflected projections to previously infected brain regions, such as rRPa and PaPy, known to be efferent targets of orexin fibers (Peyron et al., 1998; Berthoud et al., 2005), or through the very sparse projections from PeF-LH to spinal SPNs (Llewellyn-Smith et al., 2003). PRV infection did not impair orexin synthesis and expression in infected neurons since dual-labeled neurons were observed in the PeF-LH at late post-inoculation times. PRV-infected orexin neurons did not show a specific topographic distribution and were found scattered in the entire PeF-LH. Nevertheless, they were more commonly observed dorsolateral to the fornix (Fig. 1D,E) and, to a lesser extent, in the ventrolateral part of LH close to the optic tract (Fig. 1F).

At early stages of viral infection in a neuron, the virus is only detected in the nucleus and as the infection progresses the viral particles or marker ( $\beta$-gal in the case of PRV-BaBlu) are detected in both the nucleus and cytoplasm. For cell counting, neurons were considered to be infected when either the nucleus or the nucleus and cytoplasm were labeled. Similarly, dual-labeled, PRV-infected, Orx-A-ir neurons were counted when only the nucleus was infected (green nucleus, red cytoplasm) and when both nucleus and cytoplasm were infected (green nucleus, yellow cytoplasm) (Fig. $1 E$ ).

In general, the number of PRVinfected neurons (orexin and non-orexin) in PeF-LH increased at longer post-inoculation times. However, some variability in the extent of infection was found among samples within the same post-inoculation interval (this variability might be due to immobilization of the virus inside the fat, which may take longer for the viral particles to reach the axon terminals from sympathetic ganglion neurons). For this reason, we calculated the percentage of PRV-infected neurons that were orexinpositive by grouping the cases on the basis of the total number of infected neurons in the PeF-LH rather than strictly by postinoculation time. Since there were no differences in the pattern and extent of infection between PRV-Bartha- and PRV-BaBluinfected animals, these cases were grouped together. The cell counting results for the following groups are reported in Table 1: light infection (rats with $<10 \mathrm{PRV}$-infected neurons in PeF-LH, $n=5$ ), intermediate infection (rats with 10-65 PRV-infected neurons in PeF-LH, $n=5$ ) and high infection (rats with 120-180 PRV-infected neurons in PeF-LH, $n=3$ ).

The variability among cases in the light and intermediate infection groups was high, most likely because of the lower total number of infected neurons and because of a sampling effect (we counted neurons in a 1:8 set of sections). In contrast, the variability was very low in the highly infected group, suggesting that the percentage obtained $(\sim 20 \%)$ might be a good estimate of the real percentage of orexin neurons with respect to total PeF-LH neurons that are involved in the central control of sympathetic out- 
flow to BAT. This is in agreement with a report (Oldfield et al., 2002) that after PRV injection into BAT, $32 \%$ of PRVinfected neurons in the LH were orexinpositive, although direct comparison with our results is precluded by the absence of information on post-inoculation times and on the number of rats and sections per rat that were quantified in their analysis.

\section{$\mathrm{CTb}$ injection into rRPa and $\mathrm{PaPy}$}

To determine the distribution of the orexinergic neurons in PeF-LH projecting specifically to the rRPa and to the PaPy, we injected the retrograde tracer, $\mathrm{CTb}$ into either $\mathrm{rRPa}$ or $\mathrm{PaPy}$ and then examined the PeF-LH for neurons containing CTb and Orx-A. Almost all of the cells that were double-labeled for CTb and Orx-A (Fig. 2Aii) following nanoinjection of $\mathrm{CTb}$ into the rRPa (Fig. 2 Aiii) were found in the PeF-LH region between bregma: -3.0 and $-3.5 \mathrm{~mm}$ (Fig. $2 \mathrm{Ai}$ ). Overall, the distribution of double-labeled, CTband Orx-A-ir neurons (Fig. 2 Bii) following $\mathrm{CTb}$ injection in $\mathrm{PaPy}$ (Fig. 2 Biii) was similar to that following CTb injection into the rRPa: most of the Orx-A-ir neurons projecting to $\mathrm{PaPy}$ were found in the PeF-LH at levels between bregma: -3.0 and $-3.5 \mathrm{~mm}$ (Fig. 2 $\mathrm{Bi}$ ) and they were distributed both ipsilaterally and contralaterally to the injected PaPy. Whereas the double-labeled neurons in PeF-LH following $\mathrm{CTb}$ injection into the midline rPRa were evenly distributed bilaterally and rostrocaudally, those following CTb injection into the PaPy were distributed relatively more on the side ipsilateral to the injected $\mathrm{PaPy}$ and more rostrally in the PeF-LH (i.e., section bregma: $-3.00 \mathrm{~mm}$, Fig. $2 \mathrm{Bi}$ ). Also noteworthy, is the similarity in the retrograde labeling from the two medullary injection sites of non-Orx-A-ir neurons in the dorsomedial hypothalamus $(\mathrm{DMH})$ and dorsal hypothalamic area (compare localization of CTb-only neurons in bregma -3.24 sections in Fig. 2 Aii,Bii). There was also a relative absence of retrogradely labeled, non-Orx-A-ir neurons in the caudal level of the PeF-LH (section at bregma -3.48) following $\mathrm{CTb}$ injections in $\mathrm{PaPy}$ compared with the larger number of such neurons in the PeF-LH at this level following $\mathrm{CTb}$ injection into $\mathrm{rRPa}$.

\section{Injection of Orx-A into rRPa and or PaPy}

To assess the potential functional significance of the finding of a dense network of varicose orexin fibers surrounding neurons in $\mathrm{rRPa}$ and PaPy infected following PRV injections into BAT (Fig. $1 A-C)$, we determined the effect of nanoinjection of Orx-A in rRPa or PaPy on BAT SNA and BAT thermogenesis. Experiments were performed under two conditions: first, $\mathrm{T}_{\text {Core }}$ was maintained at levels, always $<37^{\circ} \mathrm{C}$, that produced a low level of baseline BAT SNA and second, $\mathrm{T}_{\text {Core }}$ was maintained at levels, usually $>37^{\circ} \mathrm{C}$, that eliminated any baseline BAT SNA. Modest skin and core cooling $\left(\mathrm{T}_{\text {Core }}: 35.9 \pm 0.1^{\circ} \mathrm{C}\right)$ produced a level of BAT SNA that was $257 \pm 35 \%$ of the control level recorded when the rats were warmed and BAT SNA was absent. Under these cool conditions, nanoinjection of Orx-A (12pmol) into $\mathrm{rRPa}$ (Fig. 3C,D) produced a rapid, strong and sustained $(>1 \mathrm{~h})$ increase in BAT SNA and in BAT thermogenesis (Fig. $3 A, B$ ). BAT SNA increased $(p<0.05, n=6)$ by $95 \%$, from $257 \pm 35 \%$ of control before Orx-A nanoinjection to a peak of $500 \pm 87 \%$ of control at $30 \mathrm{~min}$ following Orx-A nanoinjection into $\mathrm{rRPa}$, and produced a rise $(p<0.05 ; n=7)$ in $\mathrm{T}_{\mathrm{BAT}}$ from $31.9 \pm 0.4^{\circ} \mathrm{C}$ at baseline to a peak of $33.7 \pm 0.5^{\circ} \mathrm{C}$. The Orx-A-evoked stimulation of BAT thermogenesis contributed to an increase $(p<0.05 ; n=7)$ in expired $\mathrm{CO}_{2}$ from $3.0 \pm 0.1 \%$ at baseline to a peak of $3.3 \pm 0.1 \%$ and in $\mathrm{T}_{\text {Core }}$ from $35.9 \pm 0.1^{\circ} \mathrm{C}$ to a peak of $36.2 \pm 0.2^{\circ} \mathrm{C}(p<0.05 ; n=$ 7). Orx-A nanoinjection into rRPa increased HR from $412 \pm 17$ bpm to a peak of $443 \pm 9 \mathrm{bpm}(p<0.05 ; n=7)$, but did not change arterial pressure (Fig. $3 A, B)$.

Under the cool conditions, nanoinjection of Orx-A into PaPy (Fig. 4C,D) elicited a similar long lasting $(>1 \mathrm{~h})$ increase $(p<$ $0.05, n=4)$ in BAT SNA from $384 \pm 162 \%$ of control at baseline to a peak of $597 \pm 127 \%$ of control (Fig. $4 B$, top trace) that resulted in a rise $(p<0.05)$ in BAT thermogenesis from a $\mathrm{T}_{\mathrm{BAT}}$ of $33.2 \pm 0.7^{\circ} \mathrm{C}$ at baseline to a peak of $34.6^{\circ} \mathrm{C} \pm 0.6$. Simultaneously, expired $\mathrm{CO}_{2}$ rose $(p<0.05)$ from $3.2 \pm 0.5 \%$ at baseline to $3.6 \pm 0.6 \%$ peak, and $\mathrm{T}_{\text {Core }}$ increased $(p<0.05)$ from $36.3 \pm 0.4^{\circ} \mathrm{C}$ at baseline to a peak of $36.7 \pm 0.4^{\circ} \mathrm{C}$. Orx-A nanoinjection into $\mathrm{PaPy}$ evoked a tachycardia in which $\mathrm{HR}$ rose $(p<0.05)$ from $444 \pm 11 \mathrm{bpm}$ at baseline to a peak of $470 \pm 9$ bpm (Fig. $4 A, B$ ). 

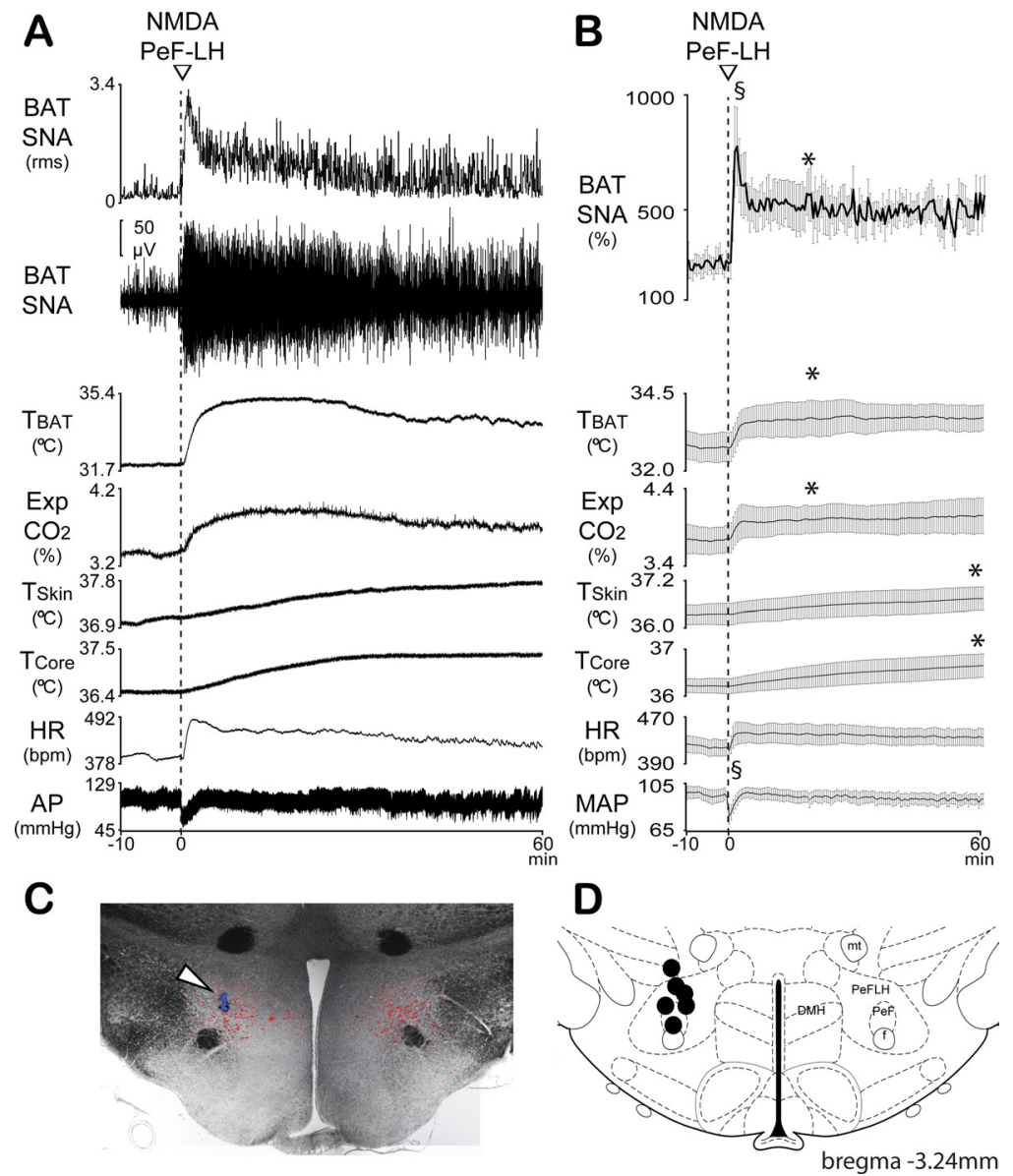

Figure 6. A, Under the baseline condition of a low level of BAT SNA, elicited by skin and core cooling, nanoinjection of NMDA (dashed line) into the PeF-LH elicited a marked and sustained $(>1 \mathrm{~h})$ enhancement of BAT SNA, $\mathrm{T}_{\mathrm{BAT}}$, and $\operatorname{expired} \mathrm{CO}_{2}\left(\operatorname{Exp} \mathrm{CO}_{2}\right)$, as well as an increase in $\mathrm{T}_{\text {Core }}$ and $\mathrm{T}_{\text {Skin. }}$. Note the early, rapidly rising period ( $\sim 3$ min duration) of elevated BAT SNA. The means \pm SEM $(n=6)$ of the time courses of the physiological variables (points are 30 s averages of the variable values) during the nanoinjection of NMDA into PeF-LH are shown in $\boldsymbol{B}$. ${ }^{\S} p<0.05$, significant increase in BAT SNA comparing the 1 min at the first peak vs 1 min window before NMDA injection into PeF-LH; ${ }^{*} p<0.05$, significant increases comparing average variable values during $1 \mathrm{~min}$ windows before NMDA injection vs $1 \mathrm{~min}$ at the peak of the sustained effect. $\boldsymbol{C}$, Histological section showing immunohistochemical labeling for orexinergic neurons (red) and the NMDA nanoinjection site (white arrowhead, blue microsphere deposit) in PeF-LH for the responses illustrated in A. D, The distribution of Orx-A nanoinjection sites (black dots) are plotted on an atlas drawing (Paxinos and Watson, 2007) containing the PeF-LH. mt, Mammillothalamic tract; MAP, mean arterial pressure; f, fornix.

\section{Injection of Orx-A into rRPa under warm conditions}

Since these data demonstrated the ability of Orx-A to potentiate an ongoing sympathetically mediated BAT thermogenesis, we next tested whether Orx-A, alone, would be sufficient to drive BAT SNA and BAT thermogenesis in the absence of basal BAT SNA. Nanoinjection of Orx-A in $\mathrm{rRPa}$ in rats whose $\mathrm{T}_{\text {Core }}$ was maintained at a level necessary to completely silence spontaneous BAT SNA (usually $37-38^{\circ} \mathrm{C}$ ). Under these warm conditions, nanoinjection of Orx-A into rRPa (Fig. $5 C, D$ ) did not elicit a change in any of the recorded variables (Fig. $5 A, B$ ). In 3 of 4 cases, several bursts of BAT SNA were present immediately following the Orx-A injection (Fig. $5 A$, second trace), however, these were sparse, short-lived and highly variable in their occurrence.

\section{Nanoinjection of NMDA into PeF-LH stimulates BAT thermogenesis}

Having demonstrated that orexin can act in the rRPa to stimulate BAT thermogenesis, that there are orexinergic neurons in
PeF-LH that are synaptically connected to BAT and that some orexinergic neurons in the PeF-LH project directly to $\mathrm{rRPa}$, we next tested the hypothesis that activation of neurons in the PeF-LH would activate BAT SNA and BAT thermogenesis in a manner similar to that evoked by Orx-A nanoinjection into $\mathrm{rPPa}$ and $\mathrm{PaPy}$. As in the experiment with Orx-A injections into $\mathrm{rRPa}$, the BAT responses to activation of neurons in PeF-LH were examined under cool conditions with a low level of ongoing BAT SNA and under warm conditions, where BAT SNA was absent. When $\mathrm{T}_{\text {Skin }}$ and $\mathrm{T}_{\text {Core }}$ were lowered to generate a low level of BAT SNA, nanoinjection of NMDA to excite local neurons, including orexin neurons, in PeF-LH (Fig. 6C,D) produced a marked and sustained increase in BAT SNA (Fig. 6A,B) that was largely similar to that following nanoinjection of Orx-A into rRPa, except for the addition of a distinct, rapid, early phase to the rise in BAT SNA (Fig. 6A,B). During this initial phase $(\sim 4$ min duration) of the increase in BAT SNA evoked by NMDA nanoinjection into PeF-LH, BAT SNA rose rapidly to a peak level that was $180 \%$ greater than that before the NMDA injection (baseline: $263 \pm 33 \%$ of control vs peak: $736 \pm 132 \%$ of control, $p<0.05$, $n=6)$ and that was significantly greater $(p<0.05, n=5$, one outlier lacking a distinct early, rapid increase in BAT SNA was excluded) than the level of BAT SNA reached during the same period following Orx-A nanoinjection into $\operatorname{rRPa}(n=6)$. There was no difference $(p>0.05)$ between the baseline levels of BAT SNA for these two groups.

After the subsidence of the early increase following NMDA nanoinjection into PeF-LH, the increase in BAT SNA was sustained for $>1 \mathrm{~h}$ and reached a peak increase of $108 \%$ (baseline: $263 \pm 33 \%$ of control vs peak: $547 \pm$ $90 \%$ of control, $p<0.05, n=6$ ), which was not different ( $p>$ 0.05 ) from the sustained increase in BAT SNA following Orx-A nanoinjection into $\mathrm{rRPa}$. The BAT thermogenic response to nanoinjection of NMDA into PeF-LH consisted of an increase in $\mathrm{T}_{\mathrm{BAT}}$ from $32.7 \pm 0.4^{\circ} \mathrm{C}$ at baseline to a peak of $33.8^{\circ} \mathrm{C} \pm 0.5(p<$ $0.05, n=6$ ), which contributed to the increase in expired $\mathrm{CO}_{2}$ (baseline: $3.7 \pm 0.2 \%$ vs peak: $4.0 \pm 0.2 \%$ peak, $p<0.05, n=6$ ) and in $\mathrm{T}_{\text {Core }}\left(36.2 \pm 0.2^{\circ} \mathrm{C}\right.$ baseline vs $36.7 \pm 0.2^{\circ} \mathrm{C}$ peak; $p<$ $0.05 ; n=6$ ) (Fig. $6 A, B$ ). Activation of neurons in PeF-LH with NMDA also evoked a brief $(\sim 2 \mathrm{~min})$ fall in arterial pressure (94 $\pm 6 \mathrm{mmHg}$ baseline vs $79 \pm 7 \mathrm{mmHg}$ nadir, $p<0.05, n=6$ ) immediately following the NMDA injection in PeF-LH (Fig. $6 A, B)$, which did not occur following Orx-A nanoinjection into rRPa (Fig. 3A, $B$ ).

\section{Injection of NMDA into PeF-LH under warm conditions}

The finding that nanoinjection of Orx-A into rRPa failed to elicit increases in BAT SNA and BAT thermogenesis in the absence of a 
basal level of ongoing BAT SNA suggests that if orexin release in the $\mathrm{rPa}$ is solely responsible for the increases in BAT SNA and BAT thermogenesis, evoked by NMDA nanoinjection into PeF-LH, then, under warm conditions, activation of neurons in PeF-LH should have no effect on BAT thermogenesis. Nanoinjection of NMDA in the PeF-LH (Fig. 7C,D) in rats whose $\mathrm{T}_{\text {Core }}$ was maintained at a level $\left(37-38^{\circ} \mathrm{C}\right)$ that completely silenced spontaneous BAT SNA, did not produce a change in any of the recorded variables (Fig. $7 A, B$ ). In 3 of 4 rats, several large bursts of BAT SNA occurred immediately after the injection of NMDA into the PeF-LH (Fig. $7 A$ ) however, as was the case following the injection of Orx-A into $\mathrm{rRPa}$ in warmed rats, these bursts were also sparse, shortlived and highly variable (Fig. $5 A, B$ ).

\section{Injection of SB-334867 in rRPa}

To establish the role of orexin receptor activation in the parallel increases in BAT SNA and BAT thermogenesis evoked by nanoinjections of Orx-A in the rRPa and of NMDA in the PeF-LH we sought to pretreat the $\mathrm{rRPa}$ with a putatively specific antagonist of the $\mathrm{Orx}_{1}-\mathrm{R}, \mathrm{SB} 334867$ under cool skin and core temperature conditions that evoked a small amount of spontaneous BAT SNA. Surprisingly, nanoinjection of SB334867 (3 nmol) into rRPa (Fig. 8C,D) produced a sustained increase in BAT SNA and BAT thermogenesis (Fig. $8 A, B$ ) that was qualitatively similar to that evoked by similar application of Orx-A in rRPa (Fig. $3 A, B$ ) or in PaPy (Fig. $4 A, B$ ), but of shorter duration than either of the latter responses (compare Fig. 8 with Figs. 3, 6). SB334867 nanoinjection into rRPa evoked a marked and rapid increase in BAT SNA (baseline: $499 \pm$ $136 \%$ of control vs peak: $1224 \pm 255 \%$ of control, $p<0.05, n=$ 5), in $\mathrm{T}_{\mathrm{BAT}}$ (baseline: $33.7 \pm 0.7^{\circ} \mathrm{C}$ vs peak: $35.4 \pm 0.7^{\circ} \mathrm{C} ; p<$ $0.05 ; n=5$ ), in expired $\mathrm{CO}_{2}$ (baseline: $3.6 \pm 0.2 \%$ vs peak: $3.9 \pm$ $0.2 \%, p<0.05, n=5$ ) and in HR (baseline: $413 \pm 10 \mathrm{bpm}$ vs peak: $442 \pm 10 \mathrm{bpm}, p<0.05, n=5$ ) (Fig. $8 A, B)$. Injection of the vehicle into $\mathrm{rRPa}$ was without effect. Even nanoinjection of a lower dose (6 pmol) of SB334867 in rRPa also caused a marked increase of BAT SNA (baseline: $235 \pm 76 \%$ of control vs peak: $624 \pm 60 \%$ of control, $p<0.05, n=3)$. In addition, nanoinjection of SB334867 (3 nmol) in rRPa following nanoinjection of Orx-A (12 pmol, $n=2)$ into rRPa or after nanoinjection of NMDA in PeF-LH (12 pmol, $n=2$ ) was without effect (data not shown) on the increased BAT SNA or BAT thermogenesis elicited by Orx-A in rRPa or activation of PeF-LH neurons.

\section{Discussion}

This study reveals a brainstem mechanism for the potent orexinergic stimulation of energy expenditure and thermogenesis in BAT which could contribute to orexin-mediated thermoregulatory and metabolic changes during a variety of behaviors, including arousal, stress and feeding. Our principal physiological findings are that (1) Orx-A increases BAT SNA and BAT thermogenesis via direct actions in the rRPa and PaPy, sites of sympathetic premotor neurons controlling BAT thermogenesis; (2) activation of neurons in the orexinergic PeF-LH increases BAT SNA and BAT thermogenesis in a manner paralleling that evoked by Orx-A in rRPa; and (3) these evoked increases in BAT thermogenesis require an ongoing, basal level of BAT SNA. These results are consistent with a role for the $\mathrm{PeF}-\mathrm{LH}$ orexinergic input to $\mathrm{rRPa}$ and $\mathrm{PaPy}$ as a gain control, amplifying the excitation of BAT sympathetic premotor neurons to enhance BAT thermogenesis. Our delineation of the precise distribution of orexin neurons that project to $\mathrm{rRPa}$ and $\mathrm{PaPy}$ provides an anatomical basis for the influence of a direct orexinergic input to the rRPa and $\mathrm{PaPy}$ on BAT thermogenesis. Indeed, the entire rostrocaudal extent of neurons in rRPa and PaPy infected following PRV injections into BAT were surrounded by a dense network of highly varicose orexin fibers. Further, orexin neurons located in the $\mathrm{PeF}-\mathrm{LH}$ became infected later than neurons in rRPa and $\mathrm{PaPy}$, some of them likely via their direct projections to infected neurons in $\mathrm{rRPa}$ and $\mathrm{PaPy}$, consistent with the retrograde labeling of orexin neurons in PeF-LH following CTb injections in $\mathrm{rRPa}$ or PaPy.

BAT thermogenesis is controlled by BAT sympathetic premotor neurons in the rRPa and PaPy. Their activation by injection of 

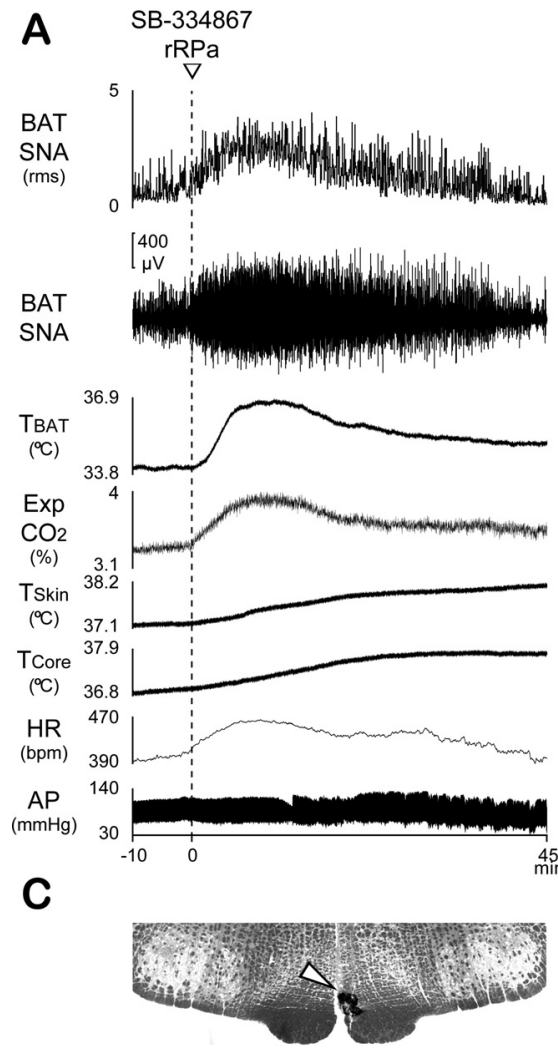

infected neurons in the PeF-LH after PRV injection into BAT and of CTb-labeled neurons in PeF-LH after CTb injection in $\mathrm{rRPa}$ and $\mathrm{PaPy}$, provides strong support for an involvement of the direct orexinergic projection to the $\mathrm{rRPa}$ and the $\mathrm{PaPy}$ in regulating BAT thermogenesis. Although putative BAT sympathetic premotor neurons in the PaPy were identified after PRV injection into BAT (Cano et al., 2003; Berthoud et al., 2005) and their role in BAT thermogenesis was proposed (Blessing, 2005), the present results provide the first direct demonstration of a physiological role for these neurons in the control of BAT thermogenesis, in this case, mediated by orexin. Injection of orexin into the $\mathrm{PaPy}$ and into the rRPa elicited similar effects on BAT thermogenesis, consistent with both populations of BAT sympathetic premotor neurons receiving a common orexinergic input from PeF-LH.

As noted in previous reports (Hermann et al., 1997; Samuels et al., 2004; Yoshida et al., 2009), our retrograde tracer injections into rRPa also labeled a distinct, non-orexinergic population of neurons in the dorsal DMH and dorsal hypothalamic area. The excitations of BAT SNA evoked from activation of neurons in the DMH (Cao et al., 2004) or in the ventrolateral LH (Cerri and Morrison, 2005) exhibited a markedly shorter time course than those evoked here by nanoinjection of NMDA into the PeF-LH or by Orx-A into the $\mathrm{rRPa}$. This observation is consistent with the unique character of the BAT thermogenic response evoked from the PeF-LH region containing orexin neurons, poten-

$\mathrm{GABA}_{\mathrm{A}}$ antagonists or excitatory amino acid agonists increases BAT SNA and thermogenesis even in animals whose $\mathrm{T}_{\text {Core }}$ is maintained at a level preventing spontaneous BAT SNA (Morrison et al., 1999; Madden and Morrison, 2003). In the present study, nanoinjection of Orx-A in the rRPa produced a strong and long-lasting increase in BAT SNA and thermogenesis only when the rats were in a slightly cooled condition that permitted spontaneous BAT SNA, but not when the rats were sufficiently warm to prevent spontaneous BAT SNA. These results suggest that orexin in the $\mathrm{rRPa}$ is not sufficient to drive BAT thermogenesis under conditions in which the excitability of BAT sympathetic premotor neurons in $\mathrm{rRPa}$ is reduced by a predominance of inhibitory inputs (Morrison et al., 1999). The specific mechanisms responsible for these observations, including the phenotypes of the $\mathrm{rRPa}$ and PaPy neurons on which orexin receptors are located, remain unknown.

We observed a dense network of orexin fibers and terminals surrounding PRV-infected neurons in the PaPy after PRV injection into BAT. In addition, Orx-A/CTb double-labeled neurons following injection of $\mathrm{CTb}$ into $\mathrm{PaPy}$ had a similar distribution throughout the PeF-LH orexinergic field as those retrogradely labeled following $\mathrm{CTb}$ injection into $\mathrm{rRPa}$. The temporal sequence of PRV infection ( $\mathrm{rRPa}$ and $\mathrm{PaPy}$ infection preceding infection in PeF-LH) and the similar distribution of PRV- tially due to a prolonged and potent modulatory effect of orexin on the excitability of BAT sympathetic premotor neurons in $\mathrm{rRPa}$. However, determining whether the amplification of BAT thermogenesis following activation of PeF-LH neurons is mediated by orexin receptors in $\mathrm{rRPa}$ must await the availability of improved orexin receptor antagonists.

Although we sought to determine whether the increased BAT thermogenesis evoked by activation of neurons in the PeF-LH was mediated by orexin release in $\mathrm{RPa}$, we found that nanoinjection of the $\mathrm{Orx}_{1} \mathrm{R}$ antagonist, $\mathrm{SB} 334867$, in $\mathrm{rRPa}$ had a strong agonist-like effect, stimulating a significant and prolonged increase in BAT SNA and BAT thermogenesis (Fig. 8). Subsequent injection of SB334867 in rRPa had no effect on the amplitudes or time courses of the increases in BAT SNA and BAT thermogenesis evoked by either Orx-A injection in rRPa or NMDA injection in PeF-LH. Consistent with our observation, SB334867 was reported to have partial agonist activity (Bengtsson et al., 2007) and chronic intracerebroventricular infusion of SB334867 acted similarly to orexin to increase BAT temperature in free-behaving rats (Verty et al., 2010). Although these observations call into question the efficacy of this drug as a selective orexin receptor antagonist, complexities of the microcircuitry in $\mathrm{rRPa}$ or the localization of orexin receptors to specific neu- 
ronal populations could explain the ability of SB334867 to activate BAT thermogenesis.

NMDA stimulation of neurons in the PeF-LH evoked an increase in BAT SNA that included an early, rapidly rising, but transient phase that was not observed in the activation of BAT SNA following Orx-A injection in rRPa (compare Figs. 3, 6). Since a significant fraction of the neurons in PeF-LH that project to rRPa are not Orx-A-ir, it may be that NMDA injection activates both the non-orexinergic population of PeF-LH neurons, resulting in a strong, initial excitation of BAT SNA, as well as the orexinergic neurons in PeF-LH, resulting in the second, longlasting activation of BAT SNA. Indeed, all the characteristics of the long-lasting response evoked from NMDA activation of PeF-LH neurons closely resemble those elicited by direct nanoinjection of orexin in $\mathrm{rRPa}$, including the long duration and the requirement for an ongoing level of basal BAT SNA. Another possibility is that this initial phase of BAT SNA excitation may arise from glutamate released from PeF-LH neurons, including $\sim 50 \%$ of the orexin neurons, that contain vesicular glutamate transporters (VGLUTs) (Rosin et al., 2003), although whether VGLUT-containing, PeF-LH neurons project to the rRPa is unknown. We could not test the glutamatergic nature of the initial BAT SNA excitation from NMDA injection in the PeF-LH since cold-evoked BAT SNA is eliminated by blockade of glutamate receptors in $\mathrm{rRPa}$ (Nakamura and Morrison, 2007). Finally, we cannot rule out an indirect pathway, since PeF-LH neurons also project to other brain regions involved in thermoregulation and metabolism, including the arcuate nucleus, median and medial preoptic areas, and the DMH (Peyron et al., 1998), that could mediate the early, transient activation of BAT SNA.

In conclusion, this study demonstrates a potent and longlasting modulatory role of orexin, acting in the $\mathrm{rRPa}$ and $\mathrm{PaPy}$, to amplify ongoing BAT sympathetic outflow, BAT thermogenesis and BAT energy expenditure. The localization of orexinergic neurons solely in the PeF-LH, the demonstration of retrogradely labeled, orexinergic neurons in PeF-LH following PRV injection into BAT and after CTb injections into rRPa and PaPy, and the finding that the BAT sympathetic and thermogenic responses to PeF-LH activation parallel those to Orx-A injection into $\mathrm{rRPa}$ and $\mathrm{PaPy}$ provide strong support for the conclusion that orexin release in the rRPa and PaPy from neurons in PeF-LH could play a significant role in setting the overall tone of BAT thermogenesis and energy expenditure and, in turn, body temperature, particularly across sleep-wake cycles, during arousal or periods of increased vigilance requiring activity or in response to dietary influences. These findings, coupled with the increasing evidence supporting an interaction between BAT energy expenditure and obesity (Hamann et al., 1998; Feldmann et al., 2009; Vijgen et al., 2011), dovetail with indications of the influence of the orexinergic system on energy expenditure from physical activity (Novak et al., 2006), to suggest that the orexinergic system is a key determinant of overall energy expenditure and thus, body weight regulation, such that a reduced orexinergic influence could increase the risk of obesity and metabolic syndrome as in narcolepsy (Poli et al., 2009), while augmented orexin activity could contribute to a lean phenotype (Funato et al., 2009).

\section{References}

Bengtsson MW, Mäkelä K, Sjöblom M, Uotila S, Akerman KE, Herzig KH, Flemström G (2007) Food-induced expression of orexin receptors in rat duodenal mucosa regulates the bicarbonate secretory response to orexin-A. Am J Physiol Gastrointest Liver Physiol 293:G501-G509.

Berthoud HR, Patterson LM, Sutton GM, Morrison C, Zheng H (2005)
Orexin inputs to caudal raphe neurons involved in thermal, cardiovascular, and gastrointestinal regulation. Histochem Cell Biol 123:147-156.

Billig I, Foris JM, Enquist LW, Card JP, Yates BJ (2000) Definition of neuronal circuitry controlling the activity of phrenic and abdominal motoneurons in the ferret using recombinant strains of pseudorabies virus. J Neurosci 20:7446-7454.

Blessing WW (2005) BAT control shows the way: medullary raphe/parapyramidal neurons and sympathetic regulation of brown adipose tissue. Am J Physiol Regul Integr Comp Physiol 288:R557-R560.

Blessing WW, Yu YH, Nalivaiko E (1999) Raphe pallidus and parapyramidal neurons regulate ear pinna vascular conductance in the rabbit. Neurosci Lett 270:33-36.

Cano G, Passerin AM, Schiltz JC, Card JP, Morrison SF, Sved AF (2003) Anatomical substrates for the central control of sympathetic outflow to interscapular adipose tissue during cold exposure. J Comp Neurol 460:303-326.

Cao WH, Morrison SF (2003) Disinhibition of rostral raphe pallidus neurons increases cardiac sympathetic nerve activity and heart rate. Brain Res 980:1-10.

Cao WH, Fan W, Morrison SF (2004) Medullary pathways mediating specific sympathetic responses to activation of dorsomedial hypothalamus. Neuroscience 126:229-240.

Card J, Enquist L (1999) Transneuronal circuit analysis with Pseudorabies virus. In: Current protocols in neuroscience, (Crawley JN et al., ed) pp 1-28. New York: Wiley.

Card JP, Enquist LW (1994) The use of neurotropic herpesviruses for defining synaptically linked populations of neurons in the central nervous system. In: Methods in molecular genetics, molecular biology techniques part A (Adolph KW, ed), pp 363-382. San Diego: Academic.

Cerri M, Morrison SF (2005) Activation of lateral hypothalamic neurons stimulates brown adipose tissue thermogenesis. Neuroscience 135:627-638.

Chen CT, Hwang LL, Chang JK, Dun NJ (2000) Pressor effects of orexins injected intracisternally and to rostral ventrolateral medulla of anesthetized rats. Am J Physiol Regul Integr Comp Physiol 278:R692-R697.

Ciriello J, Li Z, de Oliveira CV (2003) Cardioacceleratory responses to hypocretin-1 injections into rostral ventromedial medulla. Brain Res 991:84-95.

de Lecea L, Kilduff TS, Peyron C, Gao X, Foye PE, Danielson PE, Fukuhara C, Battenberg EL, Gautvik VT, Bartlett FS 2nd, Frankel WN, van den Pol AN, Bloom FE, Gautvik KM, Sutcliffe JG (1998) The hypocretins: hypothalamus-specific peptides with neuroexcitatory activity. Proc Natl Acad Sci U S A 95:322-327.

Elmquist JK, Saper CB (1996) Activation of neurons projecting to the paraventricular hypothalamic nucleus by intravenous lipopolysaccharide. J Comp Neurol 374:315-331.

Feldmann HM, Golozoubova V, Cannon B, Nedergaard J (2009) UCP1 ablation induces obesity and abolishes diet-induced thermogenesis in mice exempt from thermal stress by living at thermoneutrality. Cell Metab 9:203-209.

Funato H, Tsai AL, Willie JT, Kisanuki Y, Williams SC, Sakurai T, Yanagisawa M (2009) Enhanced orexin receptor-2 signaling prevents diet-induced obesity and improves leptin sensitivity. Cell Metab 9:64-76.

Greco MA, Shiromani PJ (2001) Hypocretin receptor protein and mRNA expression in the dorsolateral pons of rats. Brain Res Mol Brain Res $88: 176-182$.

Hamann A, Flier JS, Lowell BB (1998) Obesity after genetic ablation of brown adipose tissue. Z Ernahrungswiss 37 [Suppl 1]:1-7.

Hara J, Yanagisawa M, Sakurai T (2005) Difference in obesity phenotype between orexin-knockout mice and orexin neuron-deficient mice with same genetic background and environmental conditions. Neurosci Lett 380:239-242.

Hermann DM, Luppi PH, Peyron C, Hinckel P, Jouvet M (1997) Afferent projections to the rat nuclei raphe magnus, raphe pallidus and reticularis gigantocellularis pars alpha demonstrated by iontophoretic application of choleratoxin (subunit b). J Chem Neuroanat 13:1-21.

Kok SW, Overeem S, Visscher TL, Lammers GJ, Seidell JC, Pijl H, Meinders AE (2003) Hypocretin deficiency in narcoleptic humans is associated with abdominal obesity. Obes Res 11:1147-1154.

Kozak LP (2010) Brown fat and the myth of diet-induced thermogenesis. Cell Metab 11:263-267. 
Kuwaki T (2011) Orexin links emotional stress to autonomic functions. Auton Neurosci 161:20-27.

Llewellyn-Smith IJ, Martin CL, Marcus JN, Yanagisawa M, Minson JB, Scammell TE (2003) Orexin-immunoreactive inputs to rat sympathetic preganglionic neurons. Neurosci Lett 351:115-119.

Machado BH, Bonagamba LG, Dun SL, Kwok EH, Dun NJ (2002) Pressor response to microinjection of orexin/hypocretin into rostral ventrolateral medulla of awake rats. Regul Pept 104:75-81.

Madden CJ, Morrison SF (2003) Excitatory amino acid receptor activation in the raphe pallidus area mediates prostaglandin-evoked thermogenesis. Neuroscience 122:5-15.

Marcus JN, Aschkenasi CJ, Lee CE, Chemelli RM, Saper CB, Yanagisawa M, Elmquist JK (2001) Differential expression of orexin receptors 1 and 2 in the rat brain. J Comp Neurol 435:6-25.

Mieda M, Hasegawa E, Kisanuki YY, Sinton CM, Yanagisawa M, Sakurai T (2011) Differential roles of orexin receptor-1 and -2 in the regulation of non-REM and REM sleep. J Neurosci 31:6518-6526.

Monda M, Viggiano A, Mondola P, De Luca V (2001) Inhibition of prostaglandin synthesis reduces hyperthermic reactions induced by hypocretin1/orexin A. Brain Res 909:68-74.

Morrison SF (2011) 2010 Carl Ludwig Distinguished Lectureship of the APS Neural Control and Autonomic Regulation Section: Central neural pathways for thermoregulatory cold defense. J Appl Physiol 110:1137-1149.

Morrison SF, Nakamura K (2011) Central neural pathways for thermoregulation. Front Biosci 16:74-104.

Morrison SF, Sved AF, Passerin AM (1999) GABA-mediated inhibition of raphe pallidus neurons regulates sympathetic outflow to brown adipose tissue. Am J Physiol 276:R290-R297.

Nakamura K, Morrison SF (2007) Central efferent pathways mediating skin cooling-evoked sympathetic thermogenesis in brown adipose tissue. Am J Physiol Regul Integr Comp Physiol 292:R127-R136.

Nakamura K, Morrison SF (2008) A thermosensory pathway that controls body temperature. Nat Neurosci 11:62-71.

Nakamura K, Morrison SF (2011) Central efferent pathways for colddefensive and febrile shivering. J Physiol 589:3641-3658.

Nedergaard J, Bengtsson T, Cannon B (2011) New powers of brown fat: fighting the metabolic syndrome. Cell Metab 13:238-240.

Novak CM, Kotz CM, Levine JA (2006) Central orexin sensitivity, physical activity, and obesity in diet-induced obese and diet-resistant rats. Am J Physiol Endocrinol Metab 290:E396-E403.

Oldfield BJ, Giles ME, Watson A, Anderson C, Colvill LM, McKinley MJ (2002) The neurochemical characterisation of hypothalamic pathways projecting polysynaptically to brown adipose tissue in the rat. Neuroscience 110:515-526.

Paxinos G, Watson C (2007) The rat brain in stereotaxic coordinates, Ed 6. San Diego: Elsevier.

Peyron C, Tighe DK, van den Pol AN, de Lecea L, Heller HC, Sutcliffe JG, Kilduff TS (1998) Neurons containing hypocretin (orexin) project to multiple neuronal systems. J Neurosci 18:9996-10015.

Plazzi G, Moghadam KK, Maggi LS, Donadio V, Vetrugno R, Liguori R, Zoccoli G, Poli F, Pizza F, Pagotto U, Ferri R (2011) Autonomic disturbances in narcolepsy. Sleep Med Rev 15:187-196.
Poli F, Plazzi G, Di Dalmazi G, Ribichini D, Vicennati V, Pizza F, Mignot E, Montagna P, Pasquali R, Pagotto U (2009) Body mass indexindependent metabolic alterations in narcolepsy with cataplexy. Sleep 32:1491-1497.

Rosin DL, Weston MC, Sevigny CP, Stornetta RL, Guyenet PG (2003) Hypothalamic orexin (hypocretin) neurons express vesicular glutamate transporters VGLUT1 or VGLUT2. J Comp Neurol 465:593-603.

Sakurai T, Amemiya A, Ishii M, Matsuzaki I, Chemelli RM, Tanaka H, Williams SC, Richardson JA, Kozlowski GP, Wilson S, Arch JR, Buckingham RE, Haynes AC, Carr SA, Annan RS, McNulty DE, Liu WS, Terrett JA, Elshourbagy NA, Bergsma DJ, Yanagisawa M (1998) Orexins and orexin receptors: a family of hypothalamic neuropeptides and G proteincoupled receptors that regulate feeding behavior. Cell 92:573-585.

Sakurai T, Mieda M, Tsujino N (2010) The orexin system: roles in sleep/ wake regulation. Ann N Y Acad Sci 1200:149-161.

Samuels BC, Zaretsky DV, DiMicco JA (2004) Dorsomedial hypothalamic sites where disinhibition evokes tachycardia correlate with location of raphe-projecting neurons. Am J Physiol Regul Integr Comp Physiol 287:R472-R478.

Shiuchi T, Haque MS, Okamoto S, Inoue T, Kageyama H, Lee S, Toda C, Suzuki A, Bachman ES, Kim YB, Sakurai T, Yanagisawa M, Shioda S, Imoto K, Minokoshi Y (2009) Hypothalamic orexin stimulates feedingassociated glucose utilization in skeletal muscle via sympathetic nervous system. Cell Metab 10:466-480.

Smart D, Sabido-David C, Brough SJ, Jewitt F, Johns A, Porter RA, Jerman JC (2001) SB-334867-A: the first selective orexin-1 receptor antagonist. Br J Pharmacol 132:1179-1182.

Smith MS, True C, Grove KL (2010) The neuroendocrine basis of lactationinduced suppression of GnRH: role of kisspeptin and leptin. Brain Res 1364:139-152.

Standish A, Enquist LW, Miselis RR, Schwaber JS (1995) Dendritic morphology of cardiac related medullary neurons defined by circuit-specific infection by a recombinant pseudorabies virus expressing beta-galactosidase. J Neurovirol 1:359-368.

Trivedi P, Yu H, MacNeil DJ, Van der Ploeg LH, Guan XM (1998) Distribution of orexin receptor mRNA in the rat brain. FEBS Lett 438:71-75.

Verty AN, Allen AM, Oldfield BJ (2010) The endogenous actions of hypothalamic peptides on brown adipose tissue thermogenesis in the rat. Endocrinology 151:4236-4246.

Vijgen GH, Bouvy ND, Teule GJ, Brans B, Schrauwen P, van Marken Lichtenbelt WD (2011) Brown adipose tissue in morbidly obese subjects. PLoS One 6:e17247.

Yoshida K, Li X, Cano G, Lazarus M, Saper CB (2009) Parallel preoptic pathways for thermoregulation. J Neurosci 29:11954-11964.

Yoshimichi G, Yoshimatsu H, Masaki T, Sakata T (2001) Orexin-A regulates body temperature in coordination with arousal status. Exp Biol Med (Maywood) 226:468-476.

Zingaretti MC, Crosta F, Vitali A, Guerrieri M, Frontini A, Cannon B, Nedergaard J, Cinti S (2009) The presence of UCP1 demonstrates that metabolically active adipose tissue in the neck of adult humans truly represents brown adipose tissue. FASEB J 23:3113-3120. 